\title{
Analytical Iron Loss Evaluation in the Stator Yoke of Slotless Surface-Mounted PM Machines
}

\author{
Matteo Leandro, Student Member, IEEE, Nada Elloumi, Alberto Tessarolo, Senior Member, IEEE, \\ and Jonas Kristiansen Nøland, Member, IEEE
}

\begin{abstract}
One of the attractive benefits of slotless machines is low losses at high speeds, which could be emphasized by a careful stator core loss assessment, potentially available already at the pre-design stage. Unfortunately, mainstream iron loss estimation methods are typically implemented in the finite element analysis (FEA) environment with a constant-coefficients dummy model, leading to weak extrapolations with huge errors. In this paper, an analytical method for iron loss prediction in the stator core of slotless PM machines is derived. It is based on the extension of the 2-D field solution over the entire machine geometry. Then, the analytical solution is combined with variable- or constantcoefficient loss models (i.e., VARCO or CCM), which can be efficiently computed by vectorized post-processing. VARCO loss models are shown to be preferred at a general level. Moreover, the paper proposes a lookup-table-based (LUT) solution as an alternative approach. The main contribution lies in the numerical link between the analytical field solution and the iron loss estimate, with the aid of a code implementation of the proposed methodology. First, the models are compared against a sufficiently dense dataset available from laminations manufacturer for validation purposes. Then, all the methods are compared for the slotless machine case. Finally, the models are applied to a real case study and validated experimentally.
\end{abstract}

Index Terms-Slotless machine, analytical method, Laplace's equation, magnetic field, stator core, iron losses, rotational losses.

\section{INTRODUCTION}

$\mathbf{S}$ LOTLESS machines benefit from eliminating the slotting effect and additional related losses, as well as the cogging torque. It is an attractive low-noise solution for machines operating at ultra-high speeds. As of today, different works have been dealing with the analytical modeling of slotless machines [1]-[5]. The analytical field solution has been utilized to propose an iron loss estimate method based on constant coefficients [6]. Moreover, an extended method has been formulated with a two-component iron loss calculation in a soft magnetic composite stator core [3]. The finite iron permeability was also considered, as for SMC material, assuming infinite iron permeability would lead to a non-negligible discrepancy with respect to a real case.

In a general instance, the iron losses evaluation can be divided into two main steps. The first step is the flux density estimation in the iron parts carrying a time-varying magnetic field. To this end, the generalized field solution considering

M. Leandro and J. K. Nøland are with the Department of Electric Power Engineering, Norwegian University of Science and Technology (NTNU), Trondheim, Norway (e-mail: matteo.leandro@ntnu.no; jonas.k.noland@ntnu.no).

N. Elloumi and A. Tessarolo are with the University of Trieste, Italy.

Manuscript received August 1, 2021;
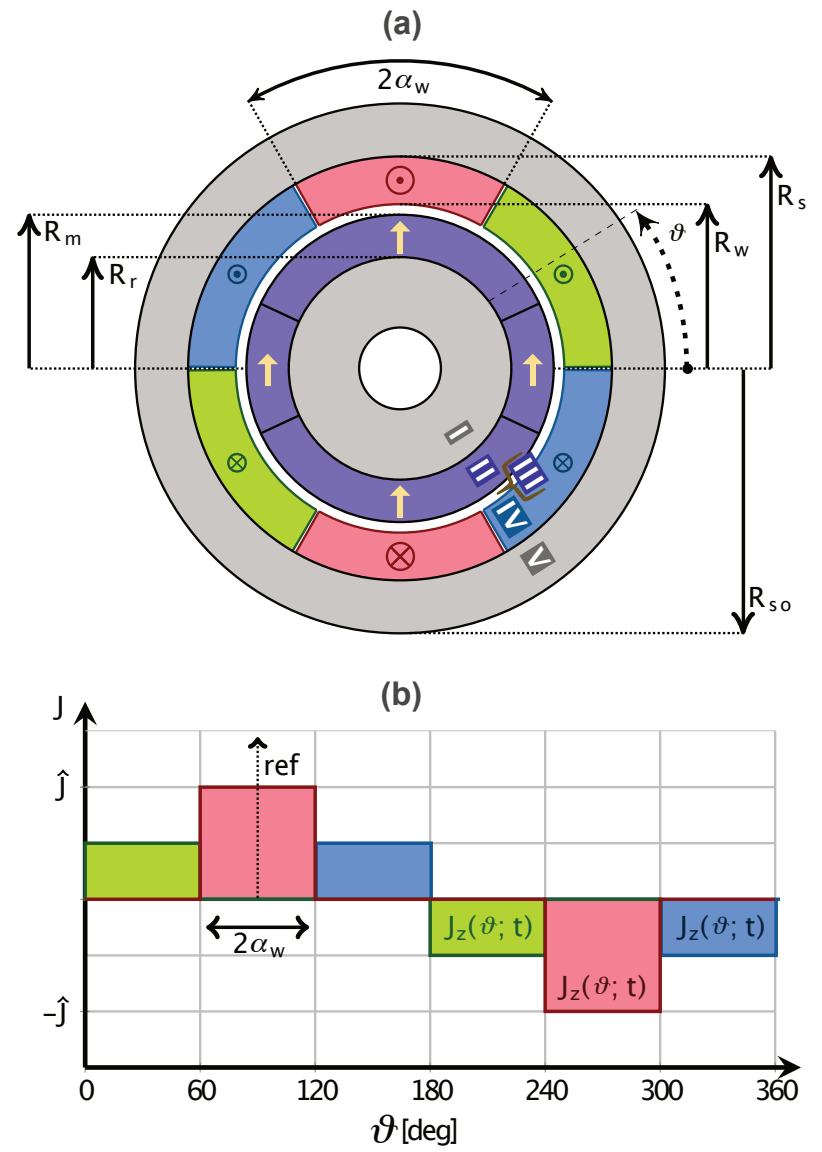

Fig. 1. (a): Generic sample geometry of the domains of an inrunner with three phases and two poles. Stator core is region V, studied hereafter. (b): Current density as a function of angular coordinate for the given time instant.

different magnet patterns will be used for a general evaluation of the flux density in the stator core. The second step is to combine the post-processing of the estimated magnetic field with a suitable model defining the losses in the iron material.

The scope of this paper is to investigate a wide range of loss models applied to the analytical field solution of slotless machines. First, the different loss modeling approaches are benchmarked against the available lamination loss dataset. Then, they are further compared on the post-processing of the analytical field solution of the slotless machine itself, assessing their performance results. Although the consolidated research in analytical modeling of electrical machines [7]-[10] and the several contributions on the development of lamination loss models [11]-[14], no concerted effort has been put on how to 
effectively merge these two topics for the sake of achieving computationally efficient design procedures. This paper extends what was presented in [15], where the link between the two topics mentioned was outlined regarding the numerical implementation. In addition, the code implementation [16] is made available, and the proposed experimental validation remarks on the applicability of the proposed methods.

The remainder of the paper is organized as follows. First, Section II presents the literature review of existing iron loss models, which will be used in the final iron loss estimate. Then, Section III derives the analytical formulation of the magnetic field in the stator core. The loss estimation methods and results are provided in Section IV. In section $V$, the advantage of the proposed methodology is highlighted. Finally, Section VI shows the experimental validation before Section VII concludes the paper.

\section{REVIEW OF LOSS Models FOR LAMINATION STEEL}

In the iron losses evaluation for electric steel laminations, Bertotti's equation represent the most adopted and widely accepted model for estimating iron losses. In the frequency domain, the different specific loss contributions can be summed up, yielding

$$
p=\underbrace{K_{e} B^{2} f^{2}}_{p_{e}}+\underbrace{K_{a} B^{1.5} f^{1.5}}_{p_{a}}+\underbrace{K_{h} B^{\alpha} f}_{p_{h}},
$$

where the three terms represent the eddy currents contribution $\left(p_{e}\right)$, the anomalous or excess loss contribution $\left(p_{a}\right)$, and the hysteresis contribution $\left(p_{h}\right)$, respectively. The different coefficients $\left(K_{e}, K_{h}, K_{a}\right.$, and $\alpha$ ) are to be determined by properly fitting the available experimental data. When dealing with FE-based iron loss analysis, the following time-based analytical formulations becomes handier with the typical transient analysis [17]-[20], where

$$
p=\underbrace{\frac{K_{e}}{2 \pi^{2}} \frac{1}{T} \int_{0}^{T}\left|\frac{d B}{d t}\right|^{2} d t}_{p_{e}}+\underbrace{\frac{K_{a}}{8.76} \frac{1}{T} \int_{0}^{T}\left|\frac{d B}{d t}\right|^{1.5} d t}_{p_{a}}+\underbrace{\frac{K_{h}}{T} B^{\alpha}}_{p_{h}}
$$

Different modified versions of the above-written equations have also been utilized. In [11], it was pointed out that the separation between eddy currents and anomalous loss contribution is questionable. Nevertheless, it is perceived that adopting a good fitting model based on only hysteresis and eddy currents contributions would inevitably include anomalous loss phenomena if any contribution exists.

The adoption of constant loss coefficients may give reasonable results when the fitting to experimental data is optimized around the most probable or rated operating condition. Thus, it can be a reasonable model when fixed speed operation and low load variation are expected. The physical phenomena governing iron losses in electrical steel laminations still represents a wide-open research topic for its inherent complexity. In fact, it is hard to imagine that a set of constant coefficients, adopted with any of the two above-mentioned models, could extensively and accurately describe such phenomena in a wide frequency and flux density range.
In [12]-[14], different loss models based on variable loss coefficients $\left(K_{h}(f, B)\right.$ and $\left.K_{e}(f, B)\right)$ are proposed, showing an improved fitting of experimental data compared to standard models based on constant coefficients (CCM). Referring to the models proposed in [11] and [12], two variable-coefficientsbased iron loss models (VARCO) and a constant-coefficientsbased model will be presented and applied to the proposed analytical method.

Another important aspect that is usually overlooked is the effect of having a rotational magnetic field when it comes to estimating iron losses. Although in some works, the assumption of having a purely alternating magnetic field is still considered [21], it appears much more reasonable to accept the rotational nature of the flux density, whereby, in the iron region, the flux density variation is determined by the simultaneous pulsation of two orthogonal flux density components ( [13], [18], [22]).

In Fig. 1 of [18], the variation of the two orthogonal flux density components is shown, for a slotted machine, in a tooth, and in the back iron. The rotational behavior of the field does not appear in the teeth. The latter may be one of the reasons why in some works, the assumption of an alternating magnetic field may lead to reasonable results, as the loss contribution from the teeth may be the most relevant one [23]. In this regard, considering slotless machines leads to the obvious conclusion that the rotational loss contribution has to be considered, being the flux density in the stator core rotational, by nature.

It has been shown experimentally, in several instances [24][27], that summing up the alternating loss contribution from the two orthogonal flux density components may lead to wrong iron loss estimates. Nonetheless, it is worth pointing out that characterizing the loss behavior of a lamination specimen requires sophisticated pieces of equipment [26], [27] which are not generally available in every research facility. Therefore, an analytical extrapolation of the data presented in Fig. 3 of reference [24] will be used, to test the impact of the rotational effect.

\section{Stator CORE AnAlytical FiEld Formulation}

The simple 2-D geometry of a slotless machine depicted in Fig. 1 enables defining accurate and computationally efficient analytical field solutions, as presented in [4], [19], [20]. Herein, the field problem is constructed upon the following fundamental assumptions.

1) The 3-D end-effects are neglected, i.e., the magnetic vector potential is treated as a scalar quantity lying orthogonal to the 2-D plane in all domains;

2) The magnetic permeability of rotor and stator core (regions I and V, respectively) is assumed to be infinite;

3) The formulation of the field from the stator current assumes the relative permeability of permanent magnets to be equal to unity;

4) For any magnets pattern, in region II the magnetization distribution is defined for modeling the field from the magnets array;

5) The stator winding is assumed to carry a three-phase balanced and symmetric sinusoidal current. 
The permanent magnet and stator current field contributions are considered separately in the definition of the final field formulation. The procedure presented herein allows defining the field solution in the whole 2D domain comprehensively. Nevertheless, the main focus will be in the iron region whereon the field solution is post-processed for the iron loss estimation procedure. The problem definition is thoroughly described in [8], with a particular focus on numerical aspects. In this work, the same field formulation is extended into the iron region, and no air gap is assumed to exist between the winding and iron yoke region.

\section{A. Stator Currents Contribution}

In a cylindrical coordinate system $(r, \vartheta, \mathrm{z})$ the formulation of the problem in the stator core region obeys to the following Laplace's equation:

$$
\nabla^{2} \mathbf{A}=\frac{1}{r} \frac{\partial}{\partial}\left[r \frac{\partial A_{z}}{\partial r}\right]+\frac{1}{r^{2}} \frac{\partial^{2} A_{z}}{\partial \theta^{2}}=0
$$

where $\boldsymbol{A}$ is the magnetic vector potential, and $A_{z}$ its only scalar component. Using the method of separation of variables along with the Fourier series expansion to account for all the harmonics introduced by the source of the field, the solution to eq. (3) can be expressed as

$$
\begin{aligned}
& A_{z, \mathrm{~V}}^{J}\left(r, \theta_{s}, t\right)= \\
& \sum_{k_{p}=5 p, 11 p, \ldots}^{\infty}\left(A_{k_{p}, \mathrm{~V}}^{+} r^{k_{p}}+A_{k_{p}, \mathrm{~V}}^{-} r^{-k_{p}}\right) \cos \left(k_{p} \theta_{s}+\omega t\right) \\
& +\sum_{k_{m}=1 p, 7 p, \ldots}^{\infty}\left(A_{k_{m}, \mathrm{~V}}^{+} r^{k_{m}}+A_{k_{m}, \mathrm{~V}}^{-} r^{-k_{m}}\right) \cos \left(k_{m} \theta_{s}-\omega t\right),
\end{aligned}
$$

where the coefficients $A_{m}^{+}$and $A_{m}^{-}$are to be determined by enforcing boundary conditions with the field solution in the winding region at the radius $R_{s}$. The flux density of the 2-D problem can be expressed as a function of the scalar magnetic potential as it follows:

$$
\begin{aligned}
& B_{r, \mathrm{~V}}^{J}\left(r, \theta_{s}\right)=\frac{1}{r} \frac{\partial A_{z, \mathrm{~V}}^{J}\left(r, \theta_{s}\right)}{\partial \theta_{s}} \\
& B_{\theta, \mathrm{V}}^{J}\left(r, \theta_{s}\right)=-\frac{\partial A_{z, \mathrm{~V}}^{J}\left(r, \theta_{s}\right)}{\partial r}
\end{aligned}
$$

By means of the latter expressions it is possible to set the following boundary conditions, that must hold for each and every harmonic component and any angular coordinate $\vartheta$ :

$$
\begin{aligned}
& \left.B_{r, \mathrm{~V}}^{J}\right|_{r=R_{s o}}=\left.\frac{1}{r} \frac{\partial A_{z, \mathrm{~V}}^{J}}{\partial \vartheta_{s}}\right|_{r=R_{s o}}=0 \\
& \left.\left(B_{r, \mathrm{~V}}^{J}=\frac{1}{r} \frac{\partial A_{z, \mathrm{~V}}^{J}}{\partial \vartheta_{s}}=B_{r, \mathrm{IV}}^{J}\right)\right|_{r=R_{s}}
\end{aligned}
$$

Boundary condition of eq. (7) is nothing more than the magnetic insulation applied to the outer boundary and justified by the infinite iron permeability assumption; whereas eq. (8) gives the continuity of the radial flux density component on the interface between stator winding $\left(B_{w r, J}\right)$ and iron core
$\left(B_{r, J}^{s t}\right)$. The boundary conditions linking the different field formulations in the different regions constitute a linear system of equations, reported here in a preconditioned matrix form as an extension of what was reported in [8]

$$
\boldsymbol{W} \boldsymbol{C}^{-1} \boldsymbol{C y}=\boldsymbol{c}
$$

where $\boldsymbol{y}$ holds the unknown terms in the different regions, $\boldsymbol{W}$ is the coefficients matrix derived from the boundary conditions, $\boldsymbol{c}$ is the right-hand-side term holding the constant terms, and $\boldsymbol{C}$ is the preconditioner as described in [8]. The different terms are reported in eqs. (10)-(13), where the different harmonic orders $\left(k_{p}, k_{m}\right)$ are merged into a single one ( $\boldsymbol{m}$ ) since eq. (9) must hold for any harmonic order. The system can be solved numerically for all the different harmonics, or symbolically. In the latter case, one can solve the system once for all, and assemble the different analytical formulations in the different regions. The formulations so obtained hold for any inrunner topology representable as in Fig. 1. It is paramount to write the field expressions with the solution coming out of the preconditioned system in eq. (9), i.e., $\boldsymbol{C} \boldsymbol{y}$ as it is numerically accurate regardless of the motor to be studied [8]. Any expression not reported here explicitly can be found in [8] as the same nomenclature is adopted.

\section{B. Permannet Magnets Field Contribution}

The magnets are modeled through their magnetization distribution in the respective region as widely done in the literature [28]-[31]. The magnetization distribution is expressed as a Fourier series along the radial and circumferential direction as follows

$$
\begin{aligned}
& M_{r}=\sum_{n=1 p, 3 p, \ldots}^{\infty} M_{r n} \cos \left(n \vartheta_{r}\right), \\
& M_{\vartheta}=\sum_{n=1 p, 3 p, \ldots}^{\infty} M_{\vartheta n} \sin \left(n \vartheta_{r}\right),
\end{aligned}
$$

where $M_{r n}$ and $M_{\vartheta n}$ are the resulting Fourier series coefficients given by the sum of the contributions of all the magnets belonging to the array to be studied. The implicit expression of the aforementioned contributions is reported in Appendix of [28] or Appendix A of [8]. Moreover, the angular coordinate $\vartheta_{r}$ is here referred to the rotating coordinates system, differently from the stator current field solution where $\vartheta_{s}$ is integral with the global coordinates system. Once the source of the field problem is modeled, the procedure described in [8], [29] can be followed to obtain the field solution due to permanent magnets in the airgap and winding regions:

$$
A_{z, \mathrm{~V}}^{M}(r, \theta)=\sum_{n=1 p, 3 p, \ldots}^{\infty}\left(A_{n, \mathrm{~V}}^{+} r^{n}+A_{n, \mathrm{~V}}^{-} r^{-n}\right) \sin \left(n \theta_{r}\right)
$$

The same boundary conditions as eqs. (7) and (8) can then be applied to the magnets field solution as follows.

$$
\left.B_{r, \mathrm{~V}}^{M}\right|_{r=R_{s e}}=\left.\frac{1}{r} \frac{\partial A_{z, \mathrm{~V}}^{M}}{\partial \vartheta_{s}}\right|_{r=R_{s e}}=0
$$




$$
\begin{gathered}
\boldsymbol{W}=\left[\begin{array}{cccccc}
R_{s o}^{m-1} & R_{s o}^{-m-1} & 0 & 0 & 0 & 0 \\
R_{s}^{m-1} & R_{s}^{-m-1} & -R_{s}^{m-1} & -R_{s}^{-m-1} & 0 & 0 \\
0 & 0 & -R_{s}^{m-1} & R_{s}^{-m-1} & 0 & 0 \\
0 & 0 & -R_{w}^{m-1} & -R_{w}^{-m-1} & R_{w}^{m-1} & R_{w}^{-m-1} \\
0 & 0 & -R_{w-1}^{m} & R_{w}^{-m-1} & R_{w}^{m-1} & -R_{w}^{-m-1} \\
0 & 0 & 0 & 0 & R_{r}^{m-1} & -R_{r}^{-m-1}
\end{array}\right] \\
\boldsymbol{c}=\left[0, A_{m}^{J} R_{s}, \frac{2 A_{m}^{J} R_{s}}{m}, A_{m}^{J} R_{w}, \frac{2 A_{m}^{J} R_{w}}{m}, 0\right]^{t} \\
\boldsymbol{C}_{I N}^{-1}= \\
\operatorname{y}=\left[A_{m, \mathrm{~V}}^{+}, A_{m, \mathrm{~V}}^{-}, A_{m, \mathrm{IV}}^{+}, A_{m, \mathrm{IV}}^{-}, A_{m, \mathrm{III}}^{+}, A_{m, \mathrm{III}}^{-}\right]^{t}\left(R_{s o}^{-m+1}, R_{s}^{m+1}, R_{s}^{-m+1}, R_{w}^{m+1}, R_{w}^{-m+1}, R_{r}^{m+1}\right)
\end{gathered}
$$$$
\left.\left(B_{r, \mathrm{~V}}^{M}=\frac{1}{r} \frac{\partial A_{z, \mathrm{~V}}^{M}}{\partial \vartheta_{s}}=B_{r, \mathrm{III}}^{M}\right)\right|_{r=R_{s}}
$$

TABLE I

SPM MACHINE DATA (COURTESy OF ALVA Industries)

These two boundary conditions allow to find the two unknowns $A_{n}^{+}$and $A_{n}^{-}$. The linear system resulting from all the boundary conditions can be written in matrix form, yielding

$$
\boldsymbol{A} \boldsymbol{P}^{-1} \boldsymbol{P} \boldsymbol{x}=\boldsymbol{b},
$$

where the coefficients matrix $\boldsymbol{A}$, the unknown vector $\boldsymbol{x}$, the vector holding the constant terms $\boldsymbol{b}$ and the preconditioner $\boldsymbol{P}$ are reported in eqs. (20)-(23), and all the terms not explicitly reported can be found in [8].

The system is solved symbolically to obtain the analytical expression of the field formulation in the whole domain. The code of the different field formulations is available from [32]. The expressions of the radial and circumferential component of the flux density in the stator core due only to the magnets' contributions are found as follows.

$$
\begin{gathered}
B_{r, \mathrm{~V}}^{M}(r, \vartheta)=\sum_{n=1 p, 3 p, \ldots}^{\infty} G_{S}(n) f_{S r}(r, n) \cos \left(n \vartheta_{r}\right) \\
B_{\vartheta, m}^{s t}(r, \vartheta)=-\sum_{n=1 p, 3 p, \ldots}^{\infty} G_{S}(n) f_{S \vartheta}(r, n) \sin \left(n \vartheta_{r}\right)
\end{gathered}
$$

The structure of the latter two flux density expressions follows the one adopted in [8], [29] which appears to be optimized for numerical implementation. The functions $G_{S}(n), f_{S r}(r, n)$ and $f_{S \vartheta}(r, n)$ are expressed, yielding

$$
\begin{aligned}
G_{S}(n) & =\frac{2 K_{B}(n)}{\left(\left(\frac{R_{s}}{R_{s o}}\right)^{2 n}-1\right)}, \\
f_{S r}(r, n) & =\left(\frac{r}{R_{s o}}\right)^{n-1}\left(\frac{R_{m}}{R_{s o}}\right)^{n+1}-\left(\frac{R_{m}}{r}\right)^{n+1}, \\
f_{S \vartheta}(r, n) & =\left(\frac{r}{R_{s o}}\right)^{n-1}\left(\frac{R_{m}}{R_{s o}}\right)^{n+1}+\left(\frac{R_{m}}{r}\right)^{n+1} .
\end{aligned}
$$

The latter field expressions have been validated already in [15]; therefore, the field comparison with FEA is here omitted.

\begin{tabular}{clc}
\hline \hline Symbol & Description & Machine specification \\
\hline$R_{r}$ & inner magnets radius & $35.1 \mathrm{~mm}$ \\
$R_{m}$ & outer magnets radius & $40.1 \mathrm{~mm}$ \\
$R_{w}$ & winding radius & $37 \mathrm{~mm}$ \\
$R_{s}$ & stator inner radius & $43.6 \mathrm{~mm}$ \\
$R_{s o}$ & stator outer radius & $44.7 \mathrm{~mm}$ \\
$l_{a}$ & active length & $30 \mathrm{~mm}$ \\
$B_{r}$ & PM remanence & $1.415 \mathrm{~T}$ \\
$\mu_{m a g}$ & PM permeability & 1.05 \\
$R_{m p}$ & mid-PM-to-pole ratio & 0.5 \\
$p$ & pole pairs & 14 \\
\hline \hline
\end{tabular}

In this work, a different case study is examined and tested experimentally. All the useful data are reported in I

The employed lamination (20JNEH1200) is a thin gauge electric steel with $0.2 \mathrm{~mm}$ thickness and a 0.97 stacking factor according to supplier's data. The supplied laminations loss data were measured according to the procedure described in [33].

\section{AnAlytically Based Iron Loss Estimation}

In the following, different iron loss models, taken from the literature, are briefly introduced and adopted with the proposed methodology to test its flexibility. The developed method will be presented as a post-processing procedure from the field solution, as obtained in the previous section, to estimate iron losses in the laminated stator core. As in [15] the armature field solution was shown not to have a significant impact on the overall iron loss, the following part will focus only on the no-load field solution, as expressed in eqs. (24), (25) and its experimental validation.

\section{A. Stator Core Discretization Method for Iron Loss Analysis}

The previous section shows that the field solution is a continuous function in the whole 2-D domain, dependent on the radial and the angular position. Based on eqs. (1) or (2), the integration of the flux density function along the iron core surface is required for evaluating iron losses. As, in a general instance, the flux density is raised to a non-integer exponent, the analytical integration along the radial direction becomes not feasible; thus, a numerical integration based on 


$$
\begin{aligned}
& \boldsymbol{A}=\left[\begin{array}{cccccc}
R_{s o}^{n-1} & R_{s o}^{-n-1} & 0 & 0 & 0 & 0 \\
R_{s}^{n-1} & R_{s}^{-n-1} & -R_{s}^{n-1} & -R_{s}^{-n-1} & 0 & 0 \\
0 & 0 & R_{s}^{n-1} & -R_{s}^{-n-1} & 0 & 0 \\
0 & 0 & \mu_{r} R_{m}^{n-1} & -\mu_{r} R_{m}^{-n-1} & -R_{m}^{n-1} & R_{m}^{-n-1} \\
0 & 0 & R_{m}^{n-1} & R_{m}^{-n-1} & -R_{m}^{n-1} & -R_{m}^{-n-1} \\
0 & 0 & 0 & 0 & \mu_{r} R_{r}^{n-1} & -\mu_{r} R_{r}^{-n-1}
\end{array}\right] \\
& \boldsymbol{x}=\left[A_{n, \mathrm{~V}}^{+}, A_{n, \mathrm{~V}}^{-}, A_{n, \mathrm{III}}^{+}, A_{n, \mathrm{III}}^{-}, A_{n, \mathrm{II}}^{+}, A_{n, \mathrm{II}}^{-}\right]^{t} \\
& \boldsymbol{b}=\left[0,0,0, \frac{A_{P M, \vartheta}\left(R_{m}\right)+\mu_{0} M_{\vartheta n}}{n} A_{P M, r}\left(R_{m}\right), \frac{A_{P M, \vartheta}\left(R_{r}\right)+\mu_{0} M_{\vartheta n}}{n}\right]^{t} \\
& \boldsymbol{P}_{I N}^{-1}=\operatorname{diag}\left(R_{s o}^{-m+1}, R_{s}^{m+1}, R_{s}^{-m+1}, R_{m}^{m+1}, R_{m}^{-m+1}, R_{r}^{m+1}\right)
\end{aligned}
$$

the rectangular rule is proposed as an effective solution for the method. In the 2-D problem, the implementation of the rectangular rule consists of dividing the iron core's radial thickness into smaller radial sub-segments whereon the flux density is assumed to be constant. Namely, in each subsegment, the flux density depends only on the rotor position. Therefore, only the integration over time or, equivalently, with respect to the stator angular coordinate, is needed; this is a simple integration of sine/cosine. Moreover, the latter operation is easily vectorizable, and thus, computationally efficient. The discretization is also shown in Fig. 2 with four sub-segments; the flux density is evaluated upon the respective mid-points.

By using the loss model described in eq. (1), the peak flux density $(B)$ becomes a matrix holding all the harmonic components in each radial sub-segment. Whereas, in eq. (2) the flux density is still a matrix containing the time-varying function (value at each time instant) on each sub-segment; thus, the differentiation can still be numerically approximated and vectorized. In particular, it is fairly easy to apply this method to the load condition operation. In fact, the magnets field variation with the rotor angular coordinate represents the time variation with respect to the static coordinate $\left(\vartheta_{s}\right)$, while for the stator current field, $\vartheta_{s}$ can be set equal to zero, and the angle variation is given by $\omega \cdot t$. Moreover, the linearity of the problem does allow to add up the two different contributions in the iron core. A flowchart of the analytical loss estimation procedure is summarized in Fig. 3. As the field solution and the iron loss estimate have been already validated through FEA in [15], the same analyses will be omitted here; however, the experimental validation will be shown instead.

\section{B. Description and Analysis of Investigated Loss Models}

The different iron loss models are briefly described in the following.

Where not explicitly specified, the losses are computed as the mere sum of iron losses due to radial and tangential components. The last case, i.e., VARCOrot will consider the rotational effect for comparison.

1) Constant Coefficients Model (CCM): The CCM uses eq. (1) for fitting all the available lamination loss data to

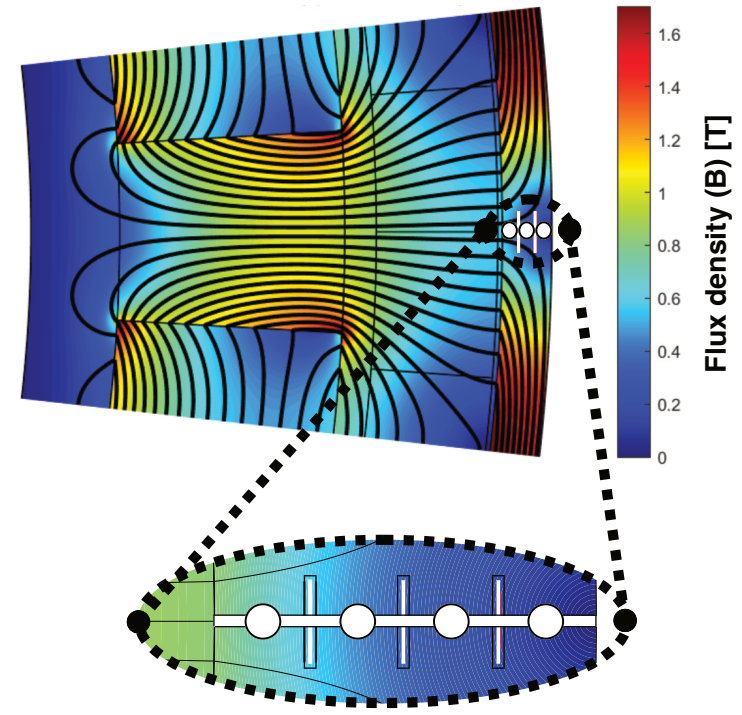

Fig. 2. Analytical field map of the case study over one pole, with the stator core discretization for integration purpose.



Fig. 3. Flowchart of the analytical loss prediction model in this paper, used for results presented in Tables II and VI.

find a general equation to be applied to any frequency and flux density value. The comparison of the lamination loss data against the proposed fitting model produced a significant relative error, which is shown in Fig. 4.

2) Two-Component Loss Model With Variable Coefficients (CAL2 [12]): The power loss density function for CAL2 is reported in [12] and expressed as

$$
p=\underbrace{k_{e}(f, B) f^{2} B^{2}}_{p_{e}}+\underbrace{k_{h}(f, B) f B^{2}}_{p_{h}},
$$




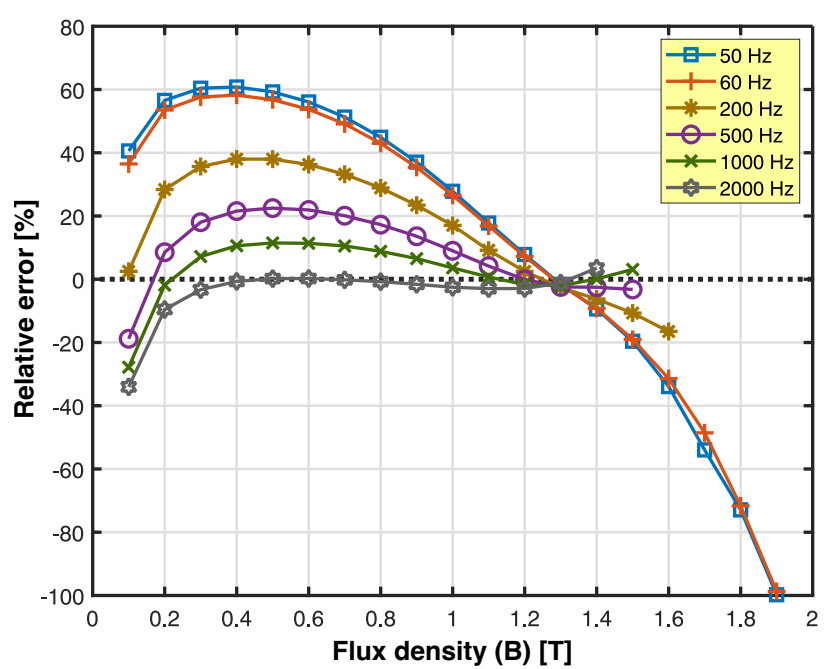

Fig. 4. Relative percentage errors of constant coefficients model (CCM) using eq. (1) (linear interpolation of available points).

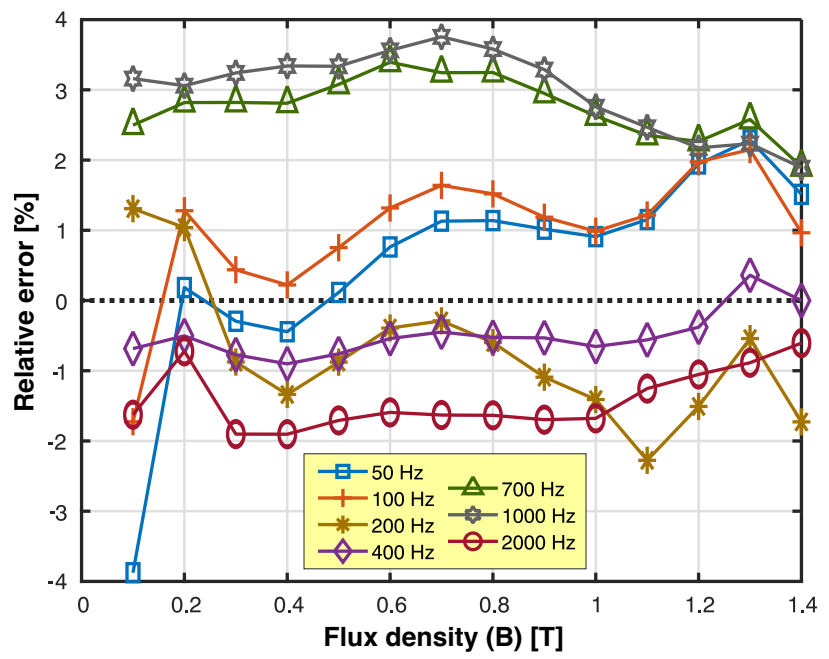

Fig. 5. Relative percentage error between experimental loss data and estimated loss with CAL2 eq. (27) iron loss model (linear interpolation between available points).

where the dependency on the frequency of the coefficients $k_{h}$ and $k_{e}$ is neglected by splitting the lamination loss data into two frequency ranges whereon the only dependency on the flux density is considered. In particular, the first range considers all the loss curves in the range $1-200 \mathrm{~Hz}$ and the second in the range $200-2000 \mathrm{~Hz}$. A sixth-order polynomial is used to express the flux density dependency of the two coefficients for both ranges. It is worth emphasizing that the model so constructed results to be the composition of two submodels where the dependency on the frequency of the different coefficients is neglected. On the "break-point" frequency, i.e., $200 \mathrm{~Hz}$ in this case, either of the two sub-models can be adopted. The first comparison between Fig. 4 and Fig. 5 shows that this type of model can be extensively used in the whole experimental data range, without any need of re-fitting the experimental data themselves when a different operating point $(f, B)$ is to be considered for the loss computation.

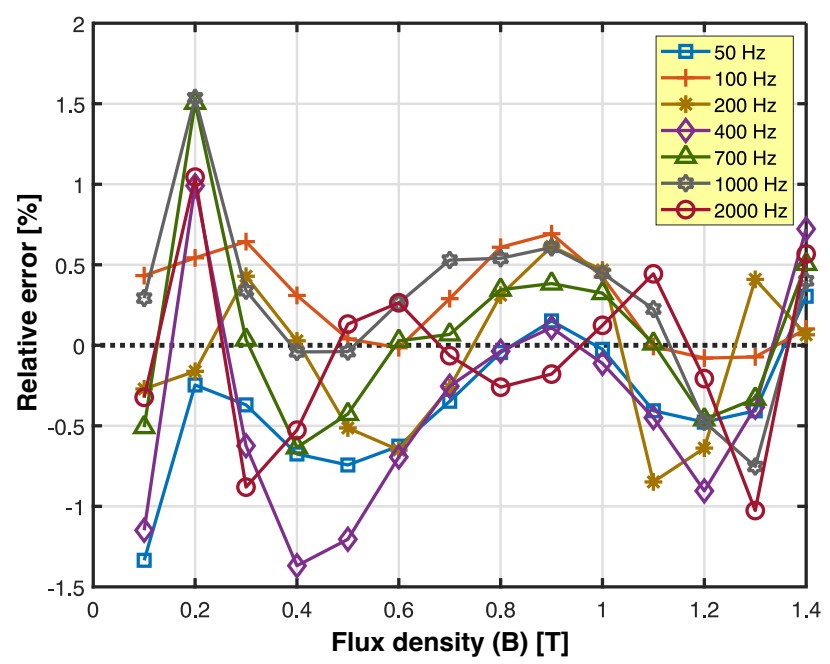

Fig. 6. Relative percentage error between experimental data and estimated loss with all variable coefficients VARCOext eq. (28) (interpolation of available points).

3) Iron Loss Model With all Variable Coefficients (VARCOext): The VARCOext model assumes the three-components loss equation having all the coefficients as variables depending on flux density and frequency, and it can be seen as an extension of what is called VARCO in [12], yielding

$p=\underbrace{k_{e}(f, B) f^{2} B^{2}}_{p_{e}}+\underbrace{k_{a}(f, B) f^{1.5} B^{1.5}}_{p_{a}}+\underbrace{k_{h}(f, B) f B^{\alpha(f, B)}}_{p_{h}}$.

The method for finding the different coefficients follows the procedure described in [11]; however, as the aim is to have an equation with explicit dependency on the frequency and flux density, the proposed representation of $\alpha(f, B)$ and $k_{h}(f, B)$, which was based on some look-up tables in [11], was here approximated by means of interpolating functions giving a reasonable fit in the two different frequency ranges $1-200 \mathrm{~Hz}$ and $200-2000 \mathrm{~Hz}$, with the "break-point" frequency at 200 $\mathrm{Hz}$. The relative error with respect to the lamination loss data produced by this loss model is represented in Fig. 6 .

4) Rotational Field Effect Appllied to VARCOext Loss Model (VARCOrot): With the aim of including the rotational effect in the iron loss computation model, VARCOext was chosen as a best-fit model for the iron losses calculation. The rotational loss factor function $(\gamma)$, can be expressed as

$$
\gamma=\frac{P_{\text {rot }}}{P\left(B_{m a j}\right)+P\left(a B_{m a j}\right)},
$$

where $a$ is the ratio between minor axis $\left(B_{\min }\right)$ and major axis $\left(B_{m a j}\right)$ component in the iron core flux density locus. From the analytical field solution, it is easy to extract major and minor axis components; therefore, the axis ratio can be found over each radial sub-segment for each harmonic component. Meaning that, yet again, an analytical function $\gamma\left(a, B_{m a j}\right)$ can be easily applied to the iron loss estimation method as a correction for the results obtained with the previous models based on summing the contribution of the two orthogonal components. The experimental results presented in Fig. 3 of reference [24] were conveniently extrapolated. However, it 
is worth mentioning that the experimental data in the same reference were obtained at $50 \mathrm{~Hz}$ fixed frequency for a specific lamination specimen which is different from the one employed in the presented case study. In fact, the purpose here is to show how flexibly such a method can be applied to the proposed methodology.

5) Look-Up-Table based Iron Loss Estimate (LUT): A different way to estimate the iron losses starting from the lamination loss data is to resort to a lookup table approach. As the main advantage, this method requires very little data post-processing when compared to the other models; besides, the computational efficiency is preserved.

The stator core discretization method can still be employed, and makes the approach very easy to develop. As already mentioned, each radial position holds a given flux density for each harmonic order. Therefore, the task is simply to locate them in the lamination loss dataset, i.e., in the lookup table (LUT), to find the loss density of each harmonic component over each radial position (see Fig. 2). There are two main paths to proceed on with the implementation. In the first case, each flux density value from the field solution is located on the closest available point in the LUT. This method can lead to a rather high inaccuracy insofar as the resolution of the data in the flux density and frequency domain is poor. In the second case, the loss density over the available data points is interpolated; in this way the field solution represents a set of scattered data points to be located onto this continuous interpolated data-set. The drawback of this approach is that it is highly sensitive to the type of interpolation, especially when the solution lies in-between two experimental data points. The LUT method can be described qualitatively by the following expression

$$
P=\rho_{f e} l_{a} \int_{S}\left[\sum_{i=1}^{\infty} p_{\text {exp,interp }}\left(f_{i},|B|_{i}\right)\right] d S
$$

where, $i$ represents a single operating point over a certain radial position, described with its flux density and frequency; the integral over the iron surface is performed numerically through the stator core discretization method described beforehand.

\section{Loss Models Comparison}

Fig. 7 shows the flowchart of the proposed procedure to validate the results of the loss models against the available dataset. It is worth mentioning that the methodology in itself is applicable to any dataset, with the only difference that the interpolating polynomials will probably have different orders depending on the dataset discretization. A dense dataset like the one used in this instance allows for smooth data interpolation from the different variable coefficient loss models.

As a quantifiable comparison between the models, the Normalized Root Mean Square Deviation (NRMSD) between lamination loss data and loss models is evaluated on the same

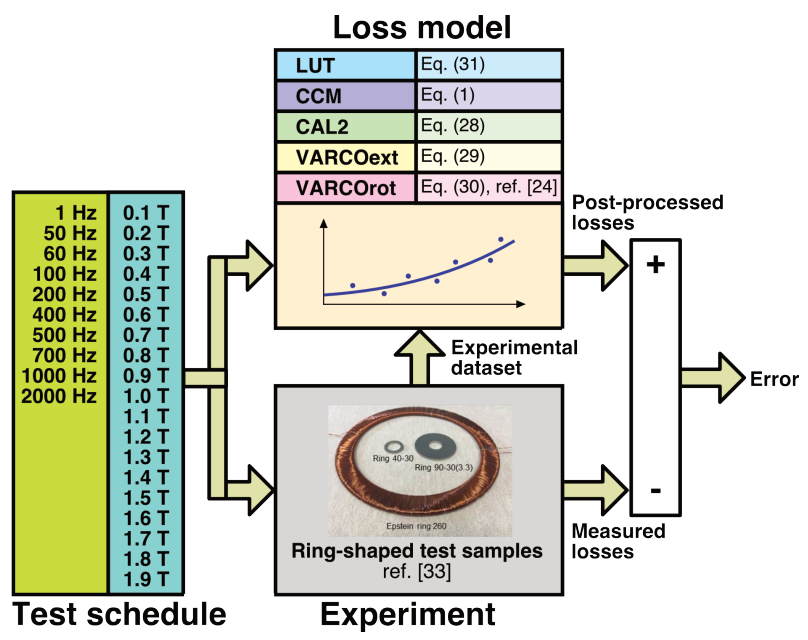

Fig. 7. Experimental data comparison for the models in this paper used for results presented in Figs. 6, 7, and 8, and Tables III, IV, and V, respectively.

TABLE II

MAXIMUM PERCENTAGE ERROR FROM EXPERIMENTAL DATA WITH DIFFERENT LOSS MODELS (ABSOLUTE VALUE)

\begin{tabular}{lcccc}
\hline \hline & CCM & CAL2 & VARCOext & LUT \\
\hline Maximum & $100 \%$ & $3.9 \%$ & $1.6 \%$ & $0 \%$ \\
percentage error & at $50 \mathrm{~Hz}$ & at $50 \mathrm{~Hz}$ & at $1000 \mathrm{~Hz}$ & (exp. \\
& and $1.9 \mathrm{~T}$ & and $0.1 \mathrm{~T}$ & and $0.2 \mathrm{~T}$ & data) \\
\hline \hline
\end{tabular}

data points as follows

$$
\begin{aligned}
R M S D & =\sqrt{\frac{\sum_{i}\left(p_{\text {mod }, i}-p_{\text {exp }, i}\right)^{2}}{N}}, \\
N R M S D & =\frac{R M S D}{p_{\text {exp }, \max }-p_{\text {exp }, \min }},
\end{aligned}
$$

where $p_{\text {exp }, i}$ and $p_{m o d, i}$ are lamination loss data, and estimated loss over the same operating point $i$; $N$ is the total number of data points within the considered range; while $p_{\exp , \max }$ and $p_{\text {exp } \text { min }}$ are the maximum and minimum loss values within the range, respectively.

In order to assess the different models, the NRMSD index is evaluated first for fixed available frequency values by sweeping over the available flux density data points; the results comparing the different loss models are reported in Table III. The same index is also evaluated at fixed available flux density values by sweeping over the available frequency data points, and the results are shown in Table III. Overall, Tables III and IV remark on what is shown in Figs. 4, 5 and 6 by means of a different index, which highlights the goodness of variable coefficients-based models; by definition, the LUT approach gives $0 \%$ error.

It is worth mentioning that the available laminations loss data-set was rather dense throughout the frequency and flux density domain (around 170 data pints). The same variable coefficients loss models applied to different and more coarse data-sets should be tuned differently, e.g., setting a different break-point frequency and using different polynomials in the two ranges. Nonetheless, the same procedure is employable. 
TABLE III

Normalized Root Mean SQuare Deviation (NRMSD) For DIFFERENT FREQUENCIES OVER THE WHOLE FLUX DENSITY SWEEP (0.1-1.6 T) FROM FIGS. 4, 5 AND 6

\begin{tabular}{ccccc}
\hline \hline Frequency & CCM & CAL2 & VARCOext & LUT \\
\hline $50 \mathrm{~Hz}$ & $30.3 \%$ & $0.77 \%$ & $0.17 \%$ & $0.00 \%$ \\
$60 \mathrm{~Hz}$ & $29.7 \%$ & $0.87 \%$ & $0.14 \%$ & $0.00 \%$ \\
$100 \mathrm{~Hz}$ & $18.4 \%$ & $0.7 \%$ & $0.11 \%$ & $0.00 \%$ \\
$400 \mathrm{~Hz}$ & $3.2 \%$ & $0.18 \%$ & $0.26 \%$ & $0.00 \%$ \\
$700 \mathrm{~Hz}$ & $1.8 \%$ & $1.06 \%$ & $0.14 \%$ & $0.00 \%$ \\
$1000 \mathrm{~Hz}$ & $1.4 \%$ & $1.04 \%$ & $0.17 \%$ & $0.00 \%$ \\
$2000 \mathrm{~Hz}$ & $1.2 \%$ & $0.54 \%$ & $0.18 \%$ & $0.00 \%$ \\
\hline
\end{tabular}

TABLE IV

Normalized Root Mean SQuare Deviation (NRMSD) For DIFFERENT FLUX DENSITIES OVER THE WHOLE FREQUENCY SWEEP (1-2000 Hz) FROM FIGS. 4, 5 AND 6

\begin{tabular}{ccccc}
\hline \hline Flux density & CCM & CAL2 & VARCOext & LUT \\
\hline $0.1 \mathrm{~T}$ & $11.3 \%$ & $0.64 \%$ & $0.13 \%$ & $0.00 \%$ \\
$0.2 \mathrm{~T}$ & $3.1 \%$ & $0.45 \%$ & $0.4 \%$ & $0.00 \%$ \\
$0.4 \mathrm{~T}$ & $2.1 \%$ & $0.73 \%$ & $0.19 \%$ & $0.00 \%$ \\
$0.5 \mathrm{~T}$ & $2.2 \%$ & $0.68 \%$ & $0.07 \%$ & $0.00 \%$ \\
$0.7 \mathrm{~T}$ & $1.9 \%$ & $0.7 \%$ & $0.06 \%$ & $0.00 \%$ \\
$0.8 \mathrm{~T}$ & $1.7 \%$ & $0.68 \%$ & $0.09 \%$ & $0.00 \%$ \\
$1.0 \mathrm{~T}$ & $1.1 \%$ & $0.63 \%$ & $0.07 \%$ & $0.00 \%$ \\
\hline \hline
\end{tabular}

\section{Flexibility of Analytical Loss Assessment}

The same iron loss models can be applied to FEA. The typical starting point is a time-domain simulation sweeping through the machine periodicity. Once the field solution is available, one can use the built-in integration operations for the time domain loss estimate. On the other hand, the procedure would be more tedious for the frequency-based loss estimate. In this case, one way of proceeding would be to load the solution on an element-basis and post-process it to transform it into the frequency domain. The frequency-based loss models can be applied on an element-by-element base; in the end, the loss contributions from all elements are summed together. This process is typically automated through routines capable of building the FEA model, solving it, and dealing with all these post-processing operations. Nevertheless, the computational time turns out to be orders of magnitudes longer than the time taken by the analytical model. In this regard, it is worth highlighting that for the analytical-based method, the angular variation of the no-load field distribution represents the time variation; this makes it possible to directly operate with the analytical expression both in the time and frequency domain. The time harmonics reflect, in fact, the space harmonics included in the field solution. Nonetheless, treating the field solution either in the time domain and frequency domain is easily vectorizable, as shown in the available code [16].

\section{Main Results And ExPERIMEnTal VALidation}

Measuring the iron loss component out of an assembled motor is undoubtedly a non-trivial challenge. In most cases, loss separation methods are used on existing motors, resorting to more or less reliable models to segregate the different loss components. These methodologies come along with several
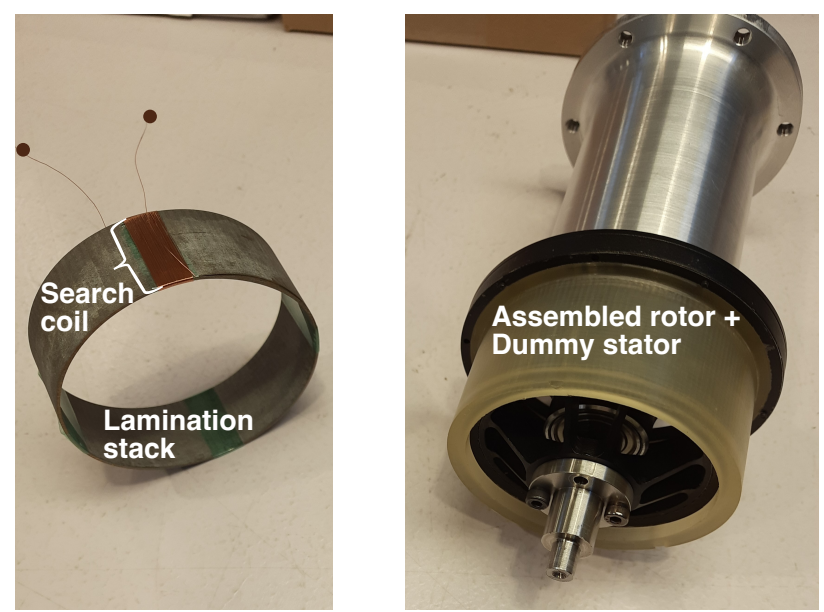

Fig. 8. Tested prototype. Assembled rotor and dummy stator on the right; laminations stack with search coil on the left.

sources of uncertainty that cannot be neglected. In this work, an existing slotless PM motor has been modified (Fig. 4) to deliver a satisfactory level of accuracy in the loss separation method. To this end, the original motor was equipped with a "dummy" non-conductive stator portion that emulates the original slotless winding to achieve the same mechanical losses in the airgap as in the real motor.

The following test procedure was adopted for the experimental validation:

1) Experimental validation of flux density on the inner stator core surface;

2) End-effect correction factor determination through induced voltage in "search coil" model and experimental measurement;

3) Iron-free prototype testing for mechanical loss measurement; and,

4) Assembled prototype testing for no-load loss measurement.

The different points are described in the following along with the obtained results.

\section{A. Experimental Field Validation}

The initial step in the experimental validation is to verify the analytical field solution used for the iron loss estimate. This was done using a gaussmeter (Hirst magnetics GM08). The probe was inserted at half the axial length for the axial leakage to be negligible between the dummy stator and the stator core, facing the latter one. The flux density was proven to be sinusoidal, and the peak flux density was measured over each pole.

In Fig. 9 the measured flux density through the gaussmeter is shown along with its error band $( \pm 0.03[T])$. The measurement showed a maximum variation of around 5\% in the peak flux density, and the estimated flux density falls into the range of each measured value, although slightly below the average. Reasons for this deviation could be one or several among mild iron saturation, end-effect due to the low axial length, Halleffect probe measuring farther in the airgap than estimated, and manufacturing tolerances. 


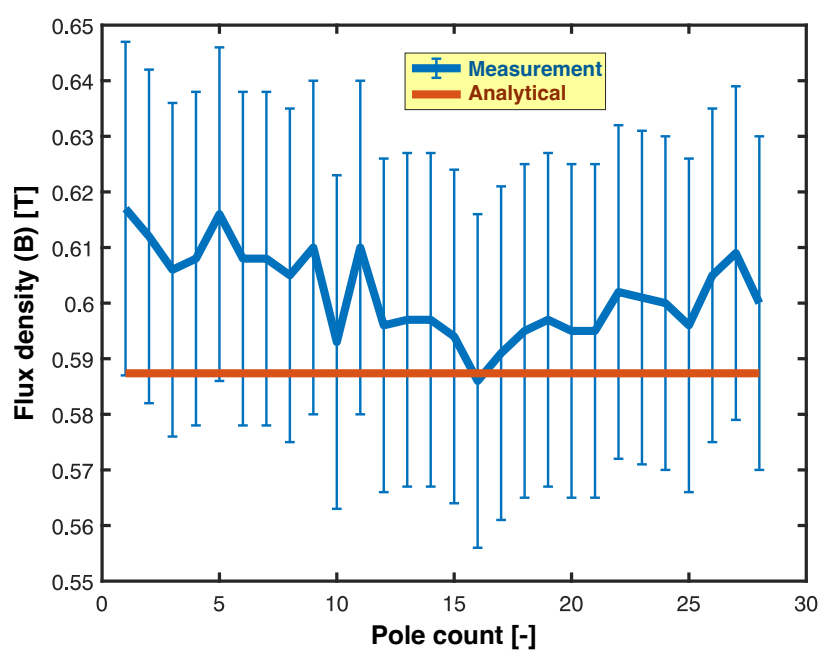

Fig. 9. Peak flux density measurement over each pole, and comparison.

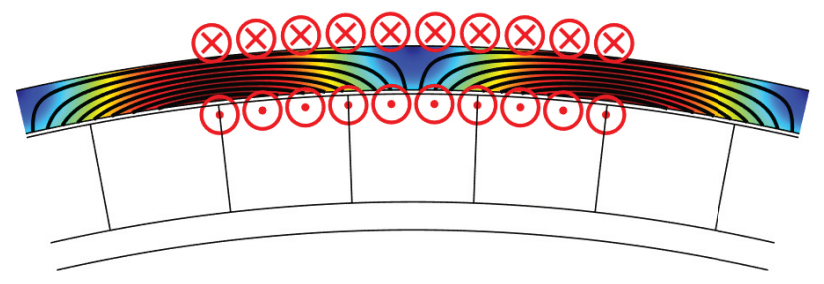

Fig. 10. Search coil representation on the $2 \mathrm{D}$ analytical field map.

\section{B. Search Coil Model and End-Effect Correction}

Proceeding with the loss analysis at this stage would inevitably produce inconsistent results, considering that a $2 \mathrm{D}$ model is being used to simulate a 3D structure. Usually, 3D FEA is employed to develop correction factors accounting for axial leakage; however, analytical alternatives do exist [34]. With the prototype available, and with the aim of validating the proposed iron loss models, an alternative experimental method has been developed. The idea is to measure the induced voltage in a coil wound around the stator core ring (left Fig. 8). The search coil can be represented in the analytical 2D field solution (Fig. 10). To find the analytical expression of the induced voltage, the coil is assumed to have negligible radial thickness; this condition is nearly met in reality as a $0.14 \mathrm{~mm}$ Litz wire strand was used.

Without reporting the intermediate steps, the analytical expression of the induced voltage is expressed as

$$
\begin{aligned}
E_{\text {coil }}= & N_{t} \frac{1}{\Delta \vartheta} R_{m} \sum_{n}\left[\left(\frac{R_{s}}{R_{s e}}\right)^{n}\left(\frac{R_{m}}{R_{s e}}\right)^{n}-\left(\frac{R_{m}}{R_{s}}\right)^{n}\right] . \\
& \cdot \frac{G_{s}(n)}{n} l_{a} \omega_{r}\left\{-\sin \left(n \omega_{r} t\right)-\sin \left[n\left(\Delta \vartheta-\omega_{r} t\right)\right]\right\}
\end{aligned}
$$

where $\Delta \vartheta$ is the angle covered by the coil, $N_{t}$ is the number of turns, and $\omega_{r}$ is the mechanical angular frequency. The coil was distributed over one pole pitch to maximize the net flux linkage. The resulting number of turns $\left(N_{t}=61.5\right)$ and the angle of the actual coil $(\Delta \vartheta 0.2351 \mathrm{rad})$ were plugged into eq. (32). The induced voltage was measured from 500 to $3500 \mathrm{rpm}$ with steps of $500 \mathrm{rpm}$. For each speed, the voltage waveform acquisition was performed so that all the poles were captured. The average peak voltage was then compared with the estimated voltage, to give the correction factor as $E_{\text {coil,peak }} / E_{\text {meas,peak }}$. This operation repeatedly produced a consistent value of 0.82 to be multiplied by the flux density fed into the iron loss models.

\section{Mechanical Loss Measurement}

For the mechanical loss measurement, the iron-free prototype shown in (Fig. 8) was tested. Any conductive part in the testbed was far enough from the magnets, so any interaction could be neglected. The braking torque was measured from a standard back-to-back setup with the supplied motor driving the tested prototype. The torque is measured through a contactless torque sensor (DR-3000, $2 \mathrm{Nm}$ rated torque, 0.05 accuracy class) in the speed range 500-3500 rpm with steps of 500 $\mathrm{rpm}$. A wide acquisition window was taken over each speed to minimize the impact of vibrations and speed instability, especially in the higher speed range. Furthermore, two sets of measurements were taken for each speed to ensure better consistency of the results.

\section{Total Loss Measurement and Iron Loss Separation}

For the iron loss measurement, the stator core was placed in position around the dummy stator. The same test routine described in the previous section was performed to measure the total losses. The mechanical losses obtained at the previous step are then subtracted from the total losses, leaving the iron loss component. The same operating points are simulated analytically with all the described models by scaling the flux density with the correction factor obtained from the search coil. The results are shown in Fig. 11 in addition the NRMSD is evaluated for each model over the shown operating range, to quantify the deviation (see Table V).

The scaled flux density in the stator core has the main component ranging from 1.4 to $1.3 \mathrm{~T}$ from the inner to the outer radius, respectively. Around this flux density value, all the different models provide an acceptable fit to the lamination loss data. For this reason, the deviation between the different models is not so remarkable in Fig. 11. From the same figure, one can notice how the rotational effect in the VARCOrot model acts as a reduction factor; this finds an explanation in the data processed to obtain the function in eq. (29). In particular, the expression is extrapolated from Fig. 3 in reference [24]. In fact, in the proposed case study, the axis ratio varies between 0.3 to 0.5 with the peak flux density going from 1.3 to $1.4 \mathrm{~T}$; in this operating range, the rotational loss factor $\gamma$ acts as a reduction factor for the iron losses computed as a mere sum of the two orthogonal contributions [24]. Even though Fig. 11 suggests the different models (VARCO in particular) to be suitable candidates for iron loss estimate through analytical modeling, it is worth mentioning that the several sources of uncertainty in the proposed validation may hide some inherent inaccuracies for the proposed models themselves. As an example, if the end-effect correction factor 
TABLE V

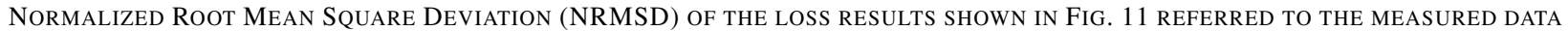

\begin{tabular}{lrrcccc}
\hline \hline & CCM & CAL2 & VARCOext & LUTlinear & LUTspline & VARCOrot \\
\hline NRMSD of Fig. 11 (Measured as ref.) & $6.73 \%$ & $8.48 \%$ & $2.33 \%$ & $6.6 \%$ & $7.4 \%$ & $8.49 \%$ \\
\hline \hline
\end{tabular}

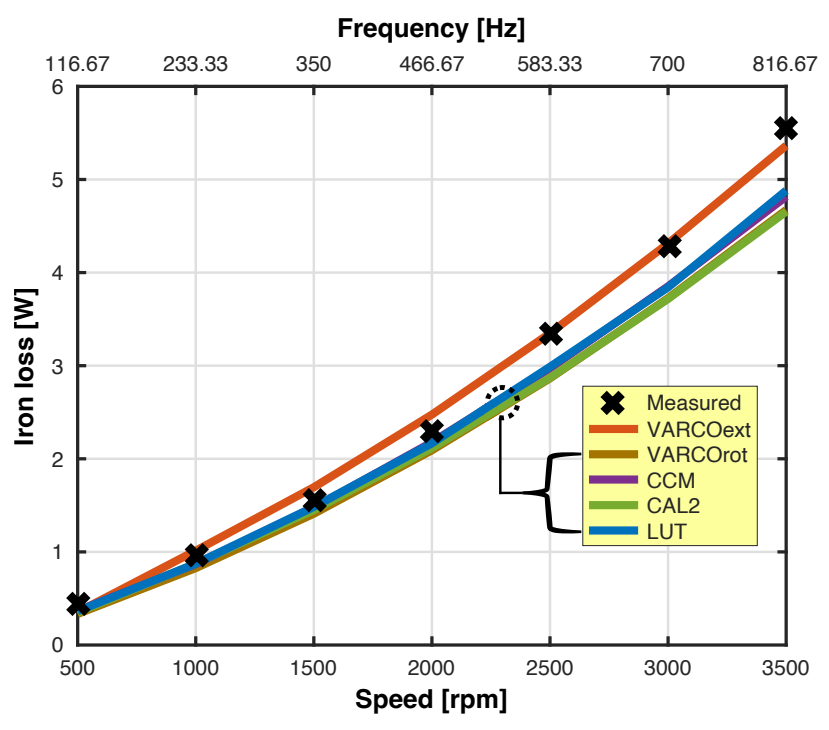

Fig. 11. Iron losses comparison between the different analytical based models and measured values on the proposed prototype (specified Table I).

was greater in reality than what was estimated, the predicted loss curves in Fig. 11 would be lifted up; in such a scenario, CCM, CAL2, LUT, VARCOrot would perform better than VARCO. To fully address the validity of the proposed loss models would require different testing prototypes to operate under different magnetic loadings to cover a broader spectrum and filter out the effect of manufacturing tolerances. Finally, the decent level of accuracy produced by the LUT methods has to be attributed to the rich available dataset; the same models may largely underperform compared to the others if a poorer dataset was available.

\section{CONCLUSION}

In this paper, the analytical field solution in the stator iron core of slotless machines is derived from Maxwell's equations. The proposed method for post-processing the field results and calculate the iron losses is described in detail. The flexibility of the method was tested by using different iron loss models, suggesting that the problem can be easily vectorized, thus, leading to a computationally efficient procedure which could be used already in a pre-design phase and/or optimization procedure. The different loss models showed that having a good fit of experimental loss data for a given specimen may be even more important than having a very accurate field solution for evaluating iron losses. In this regard, some best-fit iron loss models were proposed to overcome the latter issue. Finally, the different loss models have been benchmarked against measured losses on a test prototype.

\section{ACKNOWLEDGMENT}

The authors gratefully acknowledge the support of Alva Industries AS, especially A. Franzén, who provided assistance throughout the whole experimental validation.

\section{REFERENCES}

[1] A. Tessarolo, L. Branz, and C. Bruzzese, "A compact analytical expression for the load torque in surface permanent-magnet machines with slotless stator design," in Proc. IEEE Work. Elect. Mach. Design. Contr. Diag., March 2013, pp. 8-17.

[2] N. Bianchi, S. Bolognani, and F. Luise, "Analysis and design of a pm brushless motor for high-speed operations," IEEE Trans. Energy Convers., vol. 20, no. 3, pp. 629-637, Sep. 2005.

[3] A. Chebak, P. Viarouge, and J. Cros, "Analytical model for design of high-speed slotless brushless machines with smc stators," in Proc. IEEE Int. Elect. Mach. Drives Conf., vol. 1, May 2007, pp. 159-164.

[4] A. Tessarolo, M. Bortolozzi, and C. Bruzzese, "Explicit torque and back emf expressions for slotless surface permanent magnet machines with different magnetization patterns," IEEE Trans. Magn., vol. 52, no. 8, pp. 1-15, Aug 2016.

[5] A. Rahideh, M. Mardaneh, and T. Korakianitis, "Analytical 2-d calculations of torque, inductance, and back-emf for brushless slotless machines with surface inset magnets," IEEE Trans. Magn., vol. 49, no. 8, pp. 4873-4884, Aug 2013.

[6] L. Branz, M. Bortolozzi, and A. Tessarolo, "Analytical calculation of the no-load flux density in the stator core of slotless spm machines," in Proc. Int. Conf. Workshop Compatib. Power Electron., June 2013, pp. 244-249.

[7] H. Tiegna, Y. Amara, and G. Barakat, "Overview of analytical models of permanent magnet electrical machines for analysis and design purposes," Mathematics and Computers in Simulation, vol. 90, pp. 162-177, 2013, eLECTRIMACS 2011- PART I. [Online]. Available: https://www.sciencedirect.com/science/article/pii/S0378475413000049

[8] M. Leandro and J. K. Nøland, "An approach for optimal pre-conditioning of the analytical field solution of slotless pm machines," IEEE Access, vol. 9, pp. 36 748-36765, 2021.

[9] P. Pfister and Y. Perriard, "Slotless permanent-magnet machines: General analytical magnetic field calculation," IEEE Trans. Magn., vol. 47, no. 6, pp. 1739-1752, June 2011.

[10] B. Hannon, P. Sergeant, L. Dupré, and P.-D. Pfister, "Two-dimensional fourier-based modeling of electric machines-an overview," IEEE Transactions on Magnetics, vol. 55, no. 10, pp. 1-17, 2019.

[11] D. M. Ionel, M. Popescu, S. J. Dellinger, T. J. E. Miller, R. J. Heideman, and M. I. McGilp, "On the variation with flux and frequency of the core loss coefficients in electrical machines," IEEE Trans. Ind. Appl., vol. 42, no. 3, pp. 658-667, May 2006.

[12] D. M. Ionel, M. Popescu, M. I. McGilp, T. J. E. Miller, S. J. Dellinger, and R. J. Heideman, "Computation of core losses in electrical machines using improved models for laminated steel," IEEE Trans. Ind. Appl., vol. 43, no. 6, pp. 1554-1564, Nov 2007.

[13] J. Seo, T. Chung, C. Lee, S. Jung, and H. Jung, "Harmonic iron loss analysis of electrical machines for high-speed operation considering driving condition," IEEE Trans. Magn., vol. 45, no. 10, pp. 4656-4659, Oct 2009.

[14] M. Popescu and D. M. Ionel, "A best-fit model of power losses in cold rolled-motor lamination steel operating in a wide range of frequency and magnetization," IEEE Trans. Magn., vol. 43, no. 4, pp. 1753-1756, April 2007.

[15] M. Leandro, N. Elloumi, A. Tessarolo, and J. K. Nøland, "Analyticalbased iron loss assessment in the spm slotless machine stator core," in Proc. Int. Conf. Electr. Mach. (ICEM), 2020, pp. 772-778.

[16] M. Leandro, "Iron loss estimate from analytical field solution for inrunner slotless machines," https://www.codeocean.com/, 62021. 
[17] M. A. Mueller, S. Williamson, T. J. Flack, K. Atallah, B. Baholo, D. Howe, and P. H. Mellor, "Calculation of iron losses from time-stepped finite-element models of cage induction machines," in Proc. IEEE Int. Conf. Elect. Mach. Drives, Sep. 1995, pp. 88-.

[18] P. A. Hargreaves, B. C. Mecrow, and R. Hall, "Calculation of iron loss in electrical generators using finite element analysis," in Proc. IEEE Int. Elect. Mach. Drives Conf., May 2011, pp. 1368-1373.

[19] M. Desvaux, B. Multon, S. Sire, and H. Ben Ahmed, "Analytical iron loss model for the optimization of magnetic gear," in Proc. Int. Elect. Mach. Drives Conf., May 2017, pp. 1-8.

[20] Z. Djelloul-Khedda, K. Boughrara, F. Dubas, A. Kechroud, and A. Tikellaline, "Analytical prediction of iron-core losses in flux-modulated permanent-magnet synchronous machines," IEEE Trans. Magn., vol. 55, no. 1, pp. 1-12, Jan 2019.

[21] C. A. Hemandez-Aramburo, T. C. Green, and A. C. Smith, "Assessment of power losses of an inverter-driven induction machine with its experimental validation," in Proc. IEEE Ind. Appl. Conf. 37th IAS Ann. Meet., vol. 2, Oct 2002, pp. 1127-1134 vol.2.

[22] Lei Ma, M. Sanada, S. Morimoto, and Y. Takeda, "Prediction of iron loss in rotating machines with rotational loss included," IEEE Trans. Magn., vol. 39, no. 4, pp. 2036-2041, July 2003.

[23] K. Komeza and M. Dems, "Finite-element and analytical calculations of no-load core losses in energy-saving induction motors," IEEE Trans. Ind. Electron., vol. 59, no. 7, pp. 2934-2946, July 2012.

[24] T. Kochmann, "Relationship between rotational and alternating losses in electrical steel sheets," J. Magn. Magnet. Mater, vol. 160, pp. 145-146, 1996.

[25] C. A. Hernandez-Aramburo, T. C. Green, and A. C. Smith, "Estimating rotational iron losses in an induction machine," IEEE Trans. Magn., vol. 39, no. 6, pp. 3527-3533, Nov 2003.

[26] M. Enokizono, T. Suzuki, J. Sievert, and J. Xu, "Rotational power loss of silicon steel sheet," IEEE Trans. Magn., vol. 26, no. 5, pp. 2562-2564, Sep. 1990

[27] N. Stranges and R. D. Findlay, "Measurement of rotational iron losses in electrical sheet," IEEE Trans. Magn., vol. 36, no. 5, pp. 3457-3459, Sep. 2000.

[28] Y. Shen and Z. Zhu, "General analytical model for calculating electromagnetic performance of permanent magnet brushless machines having segmented halbach array," IET Elect. Syst. Transport., vol. 3, no. 3, pp. 57-66, Sep. 2013.

[29] Z. Q. Zhu, D. Howe, and C. C. Chan, "Improved analytical model for predicting the magnetic field distribution in brushless permanent-magnet machines," IEEE Trans. Magn, vol. 38, no. 1, pp. 229-238, Jan 2002.

[30] Y. Shen and Z. Q. Zhu, "Investigation of permanent magnet brushless machines having unequal-magnet height pole," IEEE Trans. Magn., vol. 48, no. 12, pp. 4815-4830, Dec 2012.

[31] _ _ "Analysis of electromagnetic performance of halbach pm brushless machines having mixed grade and unequal height of magnets," IEEE Trans. Magn., vol. 49, no. 4, pp. 1461-1469, April 2013.

[32] M. Leandro, "Optimal analytical field solution," https://www.codeocean.com/, 22021.

[33] A. Kahveci, P. Szary, F. Herget, A. K. Putri, and K. Hameyer, "Methods for hysteresis losses determinations at non-standard ring core geometries equivalent to epstein measurements," in 2016 6th International Electric Drives Production Conference (EDPC), 2016, pp. 135-142.

[34] Y. N. Zhilichev, "Analytic solutions of magnetic field problems in slotless permanent magnet machines," COMPEL-The international journal for computation and mathematics in electrical and electronic engineering, 2000 . 